\title{
Time variability of accretion flows: effects of the adiabatic index and gas temperature.
}

\author{
Monika Moscibrodzka ${ }^{1,2}$ and Daniel Proga ${ }^{1}$ \\ ${ }^{1}$ Department of Physics, University of Nevada, Las Vegas, NV 89154, USA, e-mail: \\ dproga@physics.unlv.edu,monikam@physics.unlv.edu \\ ${ }^{2}$ N. Copernicus Astronomical Center, Bartycka 18, 00-716, Warsaw, Poland
}

\begin{abstract}
We report on next phase of our study of rotating accretion flows onto black holes. We consider hydrodynamical (HD) accretion flows with a spherically symmetric density distribution at the outer boundary but with spherical symmetry broken by the introduction of a small, latitude-dependent angular momentum. We study accretion flows by means of numerical two-dimensional, axisymmetric, HD simulations for variety of the adiabatic index, $\gamma$ and the gas temperature at infinity, $c_{\infty}$. Our work is an extension of work done by Proga \& Begelman who consider models for only $\gamma=5 / 3$. Our main result is that the flow properties such as the topology of the sonic surface and time behavior strongly depend on $\gamma$ but little on $c_{\infty}$. In particular, for $1<\gamma<5 / 3$, the mass accretion rate shows large amplitude, slow time-variability which is a result of mixing between slow and fast rotating gas. This temporal behavior differs significantly from that in models with $\gamma \lesssim 5 / 3$ where the accretion rate is relatively constant and from that in models with $\gamma \gtrsim 1$ where the accretion exhibits small amplitude quasi-periodic oscillations. The key parameter responsible for the differences is the sound speed of the accretion flow which in turn determines whether the flow is dominated by gas pressure, radiation pressure or rotation. Despite these differences the timeaveraged mass accretion rate in units of the corresponding Bondi rate is a weak function of $\gamma$ and $c_{\infty}$.
\end{abstract}

Subject headings: accretion - hydrodynamics - black hole physics - outflows galaxies: active - methods: numerical

\section{Introduction}

Many types of astrophysical objects are powered by black hole (BH) accretion. Radiation energy produced by accretion can be very high and can explain such dramatic phe- 
nomena as quasars, powerful radio galaxies, X-ray binaries, and gamma ray bursts (GRBs). However, $\mathrm{BH}$ accretion does not always result in high radiative output. This is true for both stellar BH and super massive black holes (SMBH). In particular, objects with SMBH appear to spend statistically most of their time in an inactive phase. Inactive SMBHs are not something that one would expect, because these black holes are embedded in the relatively dense environments of galactic nuclei. Therefore it is natural to suppose that the gravity due to an SMBH will draw in matter at high rates, leading to a high system luminosity. Monitoring of X-ray binaries with stellar BHs reveals that these objects often exhibit large time variability in the total energy output in the spectral energy distribution. Then one of the main goals of any theory of $\mathrm{BH}$ accretion is two explain why accretion proceeds through very different modes.

Generally, the radiative output from accretion depends on the mass accretion rate $\dot{M}_{a}$ and an efficiency factor, $\eta$. In previous papers of this series (Proga \& Begelman 2003a,b, hereafter PB03a and PB03b, respectively), we studied how physical conditions at large distances from SMBH affect $\dot{M}_{a}$ in the so-called radiatively inefficient accretion flows (RIAF). RIAF with very low $\eta$ and also low $\dot{M}_{a}$ have been proposed to explain very low radiative luminosities in systems as such Sgr A* (e.g., Ichimaru 1977; Rees et al. 1982; Narayan \& Yi 1994, 1995; Abramowicz et al. 1995; Blandford \& Begelman 1999; Sharma et al. 2007 and reference therein). In PB03a, we addressed the issue of how $\dot{M}_{a}$ depends on the distribution of specific angular momentum, $l$ at large radii assuming that the adiabatic index, $\gamma=5 / 3$.

For high $l$, corresponding to the circularization radius larger than the last stable orbit, gas cannot directly accrete onto a BH, unless some physical mechanism like e.g., viscosity or magnetic fields, or both, transports angular momentum towards. For inviscid accretion flow the matter with too high $l$, either flows outward due to gas pressure and centrifugal forces or accumulates close the central accretor (e.g. Hawley et al. 1984a,b,; Clarke et al. 1985; Chen et al 1997; PB03a; Janiuk \& Proga 2007). The simulations presented by PB03a illustrate a general flow pattern with an inflow in the polar funnel, and an equatorial outflow (Fig. 1 there). Such flow pattern is induced by the angular momentum distribution simply because rotating gas tend to converge toward the equator. If $l$ is high, the gas forms a subsonic very dynamic torus with gas flowing out. The mass accretion rate can be significantly smaller compared to corresponding the Bondi rate.

In this work, we address the problem how the properties of the accretion flow presented in PB03a, change when different micro-physical properties of the flow are assumed. In particular, we explore effects of changing $\gamma$ in the polytropic equation of state. In reality, accretion flows with different $\gamma^{\prime}$ 's, may correspond to different types of objects or different phases of activity. For example, in the weakly active galaxies like e.g. Sgr A*, $\gamma$ is usually 
considered to be around $5 / 3$, because these flows are believed to be radiatively inefficient and gas pressure dominated. On the other hand, the large energetic output observed in GRBs indicates that an accretion flow must be radiation pressure dominated (e.g., Meszaros 2006) and a relativistic equation of state is required with $\gamma=4 / 3$. Another example are protogalactic disks, which are sometimes considered as being formed by isothermal accretion flow ( $\gamma \approx 1$; e.g., Mo et al. 1998). Also, the so-called 'high' and 'low' accretion states in the X-ray binary systems, may reflect physical proprieties changing in time (for a review see Done et al. 2007).

Our work is a straightforward extension of PB03a's work, who showed results for $\gamma=5 / 3$. We extend their models by considering the flows, for $\gamma$ ranging between 1 and 5/3. We also perform additional calculations with sound speed at infinity $c_{s, \infty}$ much smaller than that considered by PB03a allowing us to model flows with the Bondi radius as large as that estimated in real systems, for example in Sgr A*. Taking advantage of faster computers, we perform simulations with higher resolution in the $\theta$ direction, and with much larger computational domain in comparison to PB03a. We keep other model parameters such as angular momentum distribution as in PB03a.

For $\gamma=5 / 3$, our new simulations are consistent with those presented by PB03a. However, we find that $\dot{M}_{a}$, the flow structure, defined by the gas density and angular momentum distribution, and the sonic surface topology depend on $\gamma$. In particular,for $\gamma=5 / 3, \dot{M}_{a}$ is nearly constant whereas for $\gamma=4 / 3, \dot{M}_{a}$ shows stochastic, large-amplitude time-variability. For $\gamma=1.01, \dot{M}_{a}$ shows small-amplitude periodic changes. In Sec.2, we describe a general set-up of our simulations. In Sec.3, we present our results. In the last section, we discuss the results, and relate them to results found in previous studies.

\section{Method}

\subsection{Hydrodynamics}

To calculate the structure and evolution of an accreting flow, we solve the equations of hydrodynamics

$$
\begin{gathered}
\frac{D \rho}{D t}+\rho \nabla \cdot \mathbf{v}=0, \\
\rho \frac{D \mathbf{v}}{D t}=-\nabla P+\rho \nabla \Phi, \\
\rho \frac{D}{D t}\left(\frac{e}{\rho}\right)=-P \nabla \cdot \mathbf{v},
\end{gathered}
$$


where $\rho$ is the mass density, $P$ is the gas pressure, $\mathbf{v}$ is the velocity, $e$ is the internal energy density, and $\Phi$ is gravitational potential. We adopt an adiabatic equation of state $P=(\gamma-1) e$, where $\gamma$ is an adiabatic index. Our calculations are performed in spherical polar coordinates $(r, \theta, \phi)$. We assume axial symmetry about the rotational axis of the accretion flow $\left(\theta=0^{\circ}\right.$ and $\left.180^{\circ}\right)$.

We perform simulations using the pseudo-Newtonian potential $\Phi$ introduced by Paczyński \& Wiita (1980)

$$
\Phi=-\frac{G M}{r-R_{S}} .
$$

This potential approximates general relativistic effects in the inner regions, for a non-rotating black hole. In particular, the Paczyński-Wiita $(\mathrm{P}-\mathrm{W})$ potential reproduces the last stable circular orbit at $r=3 R_{S}$ as well as the marginally bound orbit at $r=2 R_{S}$.

Our standard computational domain is defined to occupy the radial range $r_{i}=1.5 R_{S} \leq$ $r \leq r_{o}=1.2 R_{B}$ and the angular range $0^{\circ} \leq \theta \leq 180^{\circ}$. We consider models with $R_{S}^{\prime}=10^{-3}$, $R_{S}^{\prime}=10^{-4}$, and $R_{S}^{\prime}=10^{-5}$. The $r-\theta$ domain is discretized into zones with 140,180 and 220 zones (for $R_{S}^{\prime}=10^{-3}, R_{S}^{\prime}=10^{-4}$, and $R_{S}^{\prime}=10^{-5}$ respectively) in the $r$ direction and 200 zones in the $\theta$ direction. We fix zone size ratios, $d r_{k+1} / d r_{k}=1.05$, and $d \theta_{l} / d \theta_{l+1}=1.0$ for $0^{\circ} \leq \theta \leq 180^{\circ}$.

\subsection{Initial conditions and boundary conditions}

For the initial conditions of the fluid variables we follow PB03a and adopt a Bondi accretion flow. In particular, we adopt $v_{r}$ and $\rho$ computed using the Bernoulli function and mass accretion rate for spherically symmetric Bondi accretion with the $\mathrm{P}-\mathrm{W}$ potential. We set $\rho_{\infty}=2.2 \times 10^{-23} \mathrm{~g} / \mathrm{cm}^{3}$ and specify $c_{s, \infty}$ through $R_{S}^{\prime} \equiv R_{S} / R_{B}$ (note that $R_{S}^{\prime}=2 c_{s, \infty}^{2} / c^{2}$, and $R_{B}=\frac{G M}{c_{s, \infty}^{2}}$ is the Bondi radius). Thus $R_{S}^{\prime}$ characterizes the gas temperature in our simulations. We specify the initial conditions by adopting a non-zero $l$ for the outer boundary of the flow.

We consider a case where the angular momentum at the outer radius $r_{o}$ depends on the polar angle via

$$
l\left(r_{o}, \theta\right)=l_{0}(1-|\cos \theta|) .
$$

We express the angular momentum on the equator as

$$
l_{0}=\sqrt{R_{C}^{\prime}} R_{B} c s_{\infty},
$$

where $R_{C}^{\prime}$ is the circularization radius on the equator in units of $R_{B}$ for the Newtonian potential (i.e., $G M / r^{2}=v_{\phi}^{2} / r$ at $r=R_{C}^{\prime} R_{B}$ ). 
The boundary conditions are specified as follows. At the poles, (i.e., $\theta=0^{\circ}$ and $180^{\circ}$ ), we apply an axis-of-symmetry boundary condition. At both the inner and outer radial boundaries, we apply an outflow boundary condition for all dynamical variables. As in $\mathrm{PB} 03 \mathrm{a}$, to represent steady conditions at the outer radial boundary, during the evolution of each model we continue to apply the constraints that in the last zone in the radial direction, $v_{\theta}=0, v_{\phi}=l(r, \theta) / r \sin \theta$, and the density is fixed at the outer boundary at all times. Note that we allow $v_{r}$ to float.

To solve eqs.( 1)-( 3) we use the ZEUS-2D code described by Stone \& Norman (1992), modified to implement the $\mathrm{P}-\mathrm{W}$ potential.

\section{Results}

Here we present results of ten simulations specified by four different values of $\gamma$ (i.e., $\gamma=5 / 3,4 / 3,1.2, \& 1.01$ ), and three values of $R_{S}^{\prime}$ (i.e., $R_{S}^{\prime}=10^{-5}, 10^{-4}$, and $10^{-3}$ ). The simulations for $\gamma=1.2$ were performed only for $R_{S}^{\prime}=10^{-3}$. Summary of all runs is presented in Tab 1. The table columns (1)-(8) show respectively the name of the run, the numerical radial resolution used in the simulation, the value of $R_{S}^{\prime}$ parameter, the value of circularization radius in units of Bondi radius $R_{C}^{\prime}, \gamma$ adiabatic index, the end time at which we stopped each simulation $t_{f}$ (the time is given in units of the dynamical time at the inner boundary $t_{d y n}=595 \mathrm{~s}$ at $\mathrm{r}=1.5 R_{S}^{\prime}$ for a mass of a black hole to be $M_{b h}=3.6 \times 10^{6} \mathrm{M}_{\odot}$ ), the maximum specific angular momentum $l_{a}^{\max }$ at the inner radial boundary, and a time-averaged value

of mass accretion rate onto a central object in units of Bondi accretion rate $\dot{M}_{a} / \dot{M}_{B}$ at the end of the simulation. To keep the same resolution at small radii for all cases, our runs with lower $R_{S}^{\prime}$, were performed with more grid points in radial direction. We followed each simulation until the quasi-stationary state was achieved, i.e., when the time averaged mass accretion rate and torus properties settled down.

\subsection{Mass accretion rate evolution}

Figs. 1, 2, and 3 show the mass accretion rate evolution, for $R_{S}^{\prime}=10^{-3}, R_{S}^{\prime}=10^{-4}$, and $R_{S}^{\prime}=10^{-5}$, respectively, for different $\gamma$. In all cases, after an episode of the spherically symmetric inflow for which $\dot{M}_{a} / \dot{M}_{B}=1, \dot{M}_{a} / \dot{M}_{B}$ decreases and starts to fluctuates around some time-averaged level. For $\gamma=5 / 3$, the mass accretion rate evolves in a similar way for all $R_{S}^{\prime}$, i.e., it stabilizes quickly and shows no strong time-variability. However, for $\gamma=4 / 3$ and $1.2, \dot{M}_{a}$ the amplitude of the fluctuations is significant. In particular, $\dot{M}_{a}$ can suddenly 
increase by a factor of $\sim 5$ and then also suddenly decrease. These flares are a result of mixing of high and low angular momentum matter (see next sections). For $\gamma=4 / 3$, the occasional flares appear for all the values of $R_{S}^{\prime}$ we explored. We suppose that the flares are also typical for flows with $\gamma=1.2$ for regardless of $R_{S}^{\prime}$.

This strong time variability for intermediate $\gamma$, is quite surprising given we explore a relatively simple HD case. Another surprising result is that the amplitude and time scale of the variability depends on $\gamma$. In particular, the flares found in models with $\gamma=4 / 3$ and 1.2 disappears in models with $\gamma=1.01$. Instead in these models, $\dot{M}_{a}$ exhibits small amplitude quasi-periodic oscillations (see next sections), for all $R_{S}^{\prime}$ values.

Comparing our results for a fixed $\gamma$ but various $R_{S}^{\prime}$, we find that the $\dot{M}_{a}$ time dependence is quite insensitive to $R_{S}^{\prime}$. This parameter determines the size of the sonic surface and therefore can somewhat affect the time-averaged $\dot{M}_{a}$ (see Table 1).

To summarize our results for $\dot{M}_{a}$, in Fig. 4 we plot the mass accretion rate at the end of the simulations as a function of $R_{S}^{\prime}$ for different $\gamma$. The final mass accretion rate is a relatively complex function of our two model parameters: for $\gamma=5 / 3, \dot{M}_{a}$ decreases with increasing $R_{S}^{\prime}$ whereas for $\gamma=1.01$ it increases with increasing $R_{S}^{\prime}$. For $\gamma=4 / 3, \dot{M}_{a}$ is not a monotonic function of $R_{S}^{\prime}$, i.e., for $R_{S}^{\prime}<10^{-3}$ it decreases with increasing $R_{S}^{\prime}$ but for $R_{S}^{\prime} \lesssim 10^{-3}$, it increases with increasing $R_{S}^{\prime}$.

As shown by PB03a, $\dot{M}_{a}$ depends on the shape and size of the sonic surface. We interpret the complex dependence of $\dot{M}_{a}$ on $\gamma$ and $R_{C}^{\prime}$ as a result of complex relation between the size of the sonic surface that depends on $R_{S}^{\prime}$ and $\gamma$ and the shape of the surface that depends strongly on $\gamma$ but does not depend significantly on $R_{S}^{\prime}$. Next, we will take a closer look at detail properties of the simulations.

\subsection{Effects of $\gamma$ and $c_{s, \infty}$ on sonic surface topology and flow properties}

Fig. 5 shows the time evolution of the direction of the poloidal velocity and contours of the sonic surfaces (i.e., where the Mach number for the poloidal velocity equals $1:\left|v_{p} / c_{s}\right|=1$ ) for four different $\gamma$ (from top to bottom, $\gamma=5 / 3,4 / 3,1.2$ and 1.01). All panels in Fig. 5 correspond to models where $R_{S}^{\prime}=10^{-3}$, and each column in the figure corresponds to the same time.

In this and the remaining part of the paper, we focus on the results obtained for $R_{S}^{\prime}=$ $10^{-3}$ (runs G, H, I, and J), because of relatively similar qualitatively results obtained for $R_{S}^{\prime} \leq 10^{-3}$. 
For $\gamma=5 / 3$ (run J, the top panels in Fig. [5), the initial spherical sonic surface, corresponding to the Bondi sonic surface, is very small $\left(r_{s} \sim 26.8 R_{S}\right)$ so that it is hard to see it in Fig. 5. Thus we refer a reader to the top right panel in Fig. 9. When the rotating gas reaches the initial sonic surface, the rotation quickly modifies the surface shape: the sphere turns into two lobes elongated along the rotational axis ("figure eight" shape). This new shape is caused by the slowdown of the gas in the equatorial region due to centrifugal force. In fact, the centrifugal force and gas pressure halt the inflow and push the gas outward along the equator. The outflowing gas creates a shock with the gas infalling from large radii. The shock then propagates outward and eventually leaves the computational domain. One can follow the evolution of the shock looking at the panels in Fig. 5 from left to right. In the right panel showing the end of the simulation, the shock has already left the computational domain. The right panel shows also that the flow lost with time its symmetry with respect to the equator (we will return to this point in Section 4).

For $\gamma=4 / 3$ (run I, the second row in Fig. 战), the sonic surface is larger than for $\gamma=5 / 3$ $\left(r_{s}=253 R_{\mathrm{s}}\right.$ instead $\left.26.8 R_{\mathrm{S}}\right)$. At the beginning of the evolution, the initially spherical sonic surface does not evolve much, because rotation at the sonic surface is slow. However, as the rotating gas reaches small radii it starts to slow down due to increasing centrifugal force. Consequently the second, inner sonic surface forms inside the original one. We refer to the initial sonic surface as the outer surface. The inner sonic surface forms because high- $l$ matter cannot accrete directly onto the central BH. In run I, gas is more gravitationally bound than in run $\mathrm{J}$ and does not flow out but rather accumulates in the inner region forming a subsonic turbulent torus. The inner flow shocks the supersonically accreting gas before the latter feels its centrifugal barrier. We define a torus as an equatorial region with high- $l$ gas and subsonic radial velocity. The torus evolves. Namely its density, gas pressure and size increase with time. Subsequently the inner sonic surface grows, too. The two sonic surfaces eventually connect as the inner surface reaches the outer sonic surface. When this happens, the accumulated matter with high $l$ starts to flow out as in run J. The sonic surface topology changes and eventually the sonic surface has the figure eight shape. We note that in run I, the figure eight shape is achieved in a different way than in run J. The second row of panels from the top in Fig. 5 show that the matter flows out, but the shock propagation is delayed in comparison run $\mathrm{J}$ (the top row of panels). At the end of run I, the flow is much more asymmetric than for run J (e.g., in run I, there is a strong outflow toward the 'north' direction).

For $\gamma=1.2$ (run H, the third row of panels in Fig. (5) the evolution of the sonic surface topology is similar to that in run I. The main difference is that the inner sonic surfaces evolves on longer time scale in run $\mathrm{H}$ than run I. The second left panel of Fig. 5 captures a moment soon after the two sonic surfaces merged. A delay in formation of the outflow is 
caused by the fact that for lower $\gamma$, the sound speed is lower and the radius of the initial outer sonic surface is larger. At the end of run $\mathrm{H}$, the flow is very asymmetric.

The simulation for $\gamma=1.01$ (run G, the bottom row of panel in Fig. 5) shows that again two sonic surfaces form as in runs $\mathrm{H}$ and I. However, the time scale for two sonic surfaces to merge is much longer in comparison to other $\gamma$ cases, because a large distant between the two surfaces (the outer surfaces is at $\sim 500 R_{S}$ ) and small sound speed. In run $\mathrm{H}$, we do not observe the formation of the outflow and we see no shock propagating outward. The time scale of the inner sonic surface growth rate is very long, and we are not able to follow it, with 200 points in $\theta$ direction, because of too long simulation time. Although not shown here, we performed a test simulation, with 2 times lower resolution in $\theta$ direction. In this test run we observe the sonic surface topology change in $\gamma=1.01$ case too, but after much longer time in comparison to other $\gamma_{\mathrm{s}}$ (e.g., the topology in run $\mathrm{G}$ changes on a time scale 150 times longer than in run $\mathrm{H}$ !). We see no outflow in the equatorial plane for this set of parameters, for very long simulation time, even after the figure eight sonic surface is formed.

We also performed additional computations for $\gamma=1.01$ models with the higher flow temperatures corresponding to $R_{S}^{\prime}=10^{-2}$ and $2 \times 10^{-2}$, but keeping the same grid parameters as for $R_{S}^{\prime}=10^{-3}$ models. As expected, in these additional runs, the flow evolves faster than in run H. In particular, an outflow forms on a reasonable time scale. Generally, we found that when we suppress the gas pressure enough by decreasing $\gamma$ or decreasing $c_{\infty}$, an outflow will take a very long time to develop. A delay in development of an outflow is a stronger function of $\gamma$ than $c_{\infty}$.

Fig. 66 compares the mass flux rates time-averaged at the end of simulations as a function of radius for runs $\mathrm{I}, \mathrm{J}, \mathrm{H}$, and $\mathrm{G}$. The mass flux rate at the given radius $\mathrm{r}$ is given by:

$$
\dot{M}=r^{2} \oint_{4 \pi} \rho v_{r} d \Omega
$$

where $d \Omega=\sin (\theta) d \theta d \phi, \rho$ is the density, and $v_{r}$ is the radial velocity. To calculate the mass inflow rate $\dot{M}_{i n}$ at a given radius, we include only contributions with $v_{r}<0$. Whereas to calculate the mass outflow rate $\dot{M}_{\text {out }}$ at a given radius, we include only contributions with $v_{r}>0$. To compute the net mass flow flux $\dot{M}_{t o t}$ we include all contributions regardless of the $v_{r}$ sign. For run J, I, and $\mathrm{H}$, the $\dot{M}_{i n}$ is larger than $\dot{M}_{\text {out }}$, and $\dot{M}_{\text {tot }}$ is constant. The latter indicates that the three models are in time-averaged steady states at the end of simulations. We do not show it here, but we check that the total mass in the computational domain does not change in time that again indicates the time-averaged steady state. On the other hand, run $\mathrm{G}$ does not settles into a steady state as matter continues to accumulate between $5 R_{S}<r<100 R_{S}$ and $\dot{M}_{t o t}$ changes with radius.

Fig. 7 compares the mass flux rates as functions of time at the outer boundary of the 
grid. We calculate $\dot{M}_{\text {in }}$ and $\dot{M}_{\text {out }}$ from eq. 7 for a given time, in the same way as shown in Fig. 6 but assuming that $\mathrm{r}=r_{o}$. For runs J, I, and H, the mass inflow rate is constant for the short time at the beginning of the simulations. The gaps in the early time of simulations are caused by the shock transition through the outer boundary. In the later times the $\dot{M}_{i n}$ and the $\dot{M}_{\text {out }}$ oscillate and are anticorrelated. For G model, there is no outflow and $\dot{M}_{\text {out }}$ and $\dot{M}_{i n}$ is constant during the whole simulation time.

Next we present and describe in more details the structure of the flow for different $\gamma$. Figs. 8, 9, and 10 show the 2-D structure of various quantities from the simulations for different $\gamma$, on different scales (i.e., $20 R_{S}, 200 R_{S}$, and $1200 R_{S}$, in Fig. 8, 9, and 10, respectively), at the end of the simulations. Each of the figures consists of four rows, in which we present results for $\gamma=5 / 3,4 / 3,1.2$ and 1.01 (panels from top to bottom, J, I, H, and $\mathrm{G}$ runs respectively). From left to right the panels show the density temperature maps, angular momentum contours, angular velocity contours and direction of the poloidal velocity overplotted by the sonic surface shape.

In all four cases, characterized by different $\gamma$ index, a torus forms. We distinguish three kinds of tori: gas pressure dominated torus, with $\gamma=5 / 3$, 'radiation pressure' dominated torus with $\gamma=4 / 3$, and for $\gamma=1.01$, due to weak dependency of pressure on density, the torus is rotation dominated.

Run $\mathrm{J}$ here corresponds to run B03f1a in PB03a. Our results are in full agreement with the previous simulations in all respects. The torus is subsonic, the angular momentum distribution in the torus is nearly constant $l \sim 0.92 R_{S} c$ (see also Fig. 11 described below). The matter accretes through the polar funnel, and flows out in the equatorial region. The mass accretion rate for $\gamma \sim 5 / 3$ and $R_{S}^{\prime}=10^{-3}$ is consistent with the previous calculations of PB03a with two times lower angular resolution.

For $\gamma=4 / 3$ (run I) the radius averaged $l$ in torus is a little higher than in $\gamma=5 / 3$ case, and it is around 1.1 of the critical value. The density gradient between polar funnels and torus is larger than in run J. The sonic surface is strongly asymmetrical. There is the above mentioned outflow in 'north' direction. Note that in Figs. 8, 9, and 10, the angular momentum contours undergo strong compression on the 'north' side of the flow. The contours are also compressed in the 'southern' part of the grid. This compression is due to the torus movement in ' $\mathrm{z}$ ' direction which we will discussed in more detail in the next subsection.

In run $\mathrm{H}$, an equatorial outflow is asymmetric and supersonic at large radii. Fig. 10, illustrates strong mixing of high- and low- $l$ matter at large distances in the 'southern' part of the grid leading to an increase in of the mass accretion rate seen as flares in Figs. 1, 2, and 3 (thick dashed lines). 
For $\gamma=1.01$ (run $\mathrm{G}$ ), the inflow is a very symmetric, at large radii. On smaller scales $\left(\mathrm{r}<200 R_{S}\right)$, the high- $l$ turbulent torus (red and yellow region in density map in Fig 9 , bottom panels) is surrounded by slightly lower density, nearly laminar inflow region (the green region in the density maps in the same panel). The high- $l$ matter flows around and supplies the matter to the turbulent torus. The torus expands in the ' $z$ ' direction with time (see Figs 10 and 12). Thus to reach the black hole, the low- $l$ matter has to flow around an growing in size an obstacle. As a result, an oblique shock, corresponding to the $v_{\theta}$ sonic surface, forms around torus. The oblique shock surrounds the above mentioned region of slightly higher density (green region in density maps). For $\mathrm{r}<20 R_{S}$ (bottom panels in Fig. 8), the torus shape remains quite flat, and is of high density and angular momentum with the latter approaching Keplerian distribution. In this inner region, the low- $l$ gas flows toward the equatorial plane almost parallel to the symmetry axis (note that the angular momentum contours outside the torus are parallel to the rotational axis). We check that the stream lines of the accreting gas, once they reach the torus they follow the torus shape.

For $\mathrm{r}<5 R_{S}$, the low-l matter forms an accretion cusp. For some time during at the initial evolution, the accretion cusp expand in the ' $z$ ' direction, because of the torus cusp grows in $\theta$ direction. This growth slightly reduces polar funnel accretion, which dominates $\dot{M}_{a}$. However soon, the size of the cusp stabilizes. The effect of cusp expansion can be seen in the accretion rate evolutionary curve dip (Fig. 1, thin solid line) around $t=2.0 \times 10^{4}$. Later the torus expansion occurs at larger radii only.

One of the most intriguing property of all the runs is that the angular velocity inside the turbulent torus, has cylindrical distribution even though the radial profile of rotation depends on $\gamma$. Fig. 11] shows radial profiles of $l$ along the equator for models with different $\gamma$ at the end of the simulations. For comparison, Keplerian $l$ distribution and $l$ corresponding to constant angular velocity are shown too.

As found by PB03a, $l$ is constant in the torus for $\gamma=5 / 3$. We find that $l$ does not change much inside the torus also for $\gamma=4 / 3$ and 1.2 though the actual value of $l$ in the torus increases with decreasing $\gamma$. As several other properties, the rotational profile for $\gamma=1.01$ differs from that for other $\gamma$. Namely, in run G, the profile is almost Keplerian for small radii (compare solid and dash-dotted line in Fig. 11).

A detailed inspection of Fig. 11] shows that $l$ sharply decreases with radius for very small radii in runs $H$ and $I$. This decrease is due to the fact that the inner flow is asymmetric and the gas reaching the $\mathrm{BH}$ at the end of these runs is not that of the torus but is of the gas with low $l$. The asymmetry also explains why $l_{\text {max }}$, listed in Table 1 , is higher than $l$ at $r_{\mathrm{i}}$ in Fig. 11, i.e., the maximum accreted $l$ does not have to and is not always at the equator. The increase of $l$ with radius at large radii is caused by the outer boundary conditions we 
use with these simulations (i.e., we reset $v_{\phi}$ at $r_{\mathrm{o}}$ to its initial value at each time step).

\subsection{Time-variability and asymmetry of the flow}

We find that the temporal behavior and sonic surface topology of the accretion flow depend on $\gamma$. In runs with $\gamma=4 / 3,1.2$, and 1.01 two sonic surfaces can exit. The sonic surface topology evolves in the similar way in all runs with the three $\gamma$ 's although on different time scales (the evolution slows down with decreasing $\gamma$ ). During the early evolution, gas with large angular momentum (larger than $l_{c r}$ ) is locked in a turbulent torus. The torus expands but there is no strong outflow because the gas motion is suppressed by supersonically infalling matter. During this phase, an oblique shock (seen as a jump in $v_{\theta}$ in Figs. 8 and 91) forms around the torus. By contrast, in runs with $\gamma=5 / 3$, only one sonic surface exists and its shape turns from initially spherical to the figure eight like one. The differences in the sonic surface topologies are reflected in the mass accretion rate curve: for runs with one sonic surface the curves are smooth whereas for runs with two sonic surfaces the curves are not smooth (compare various curves in Figs. 1, 2, and 3).

Generally, the time behavior of the flow depends mainly on the Mach number which in our simulations depends on $\gamma$ and $c_{\infty}$. To connect the time dependence of $\dot{M}_{a}$ with the flow properties we start with an analysis of run $G$ with the highest Mach number of the inner flow. In run G, $\dot{M}_{a}$ shows oscillations which one can attribute to oscillations of the inner flow in the $z$ direction (see Fig. 12 showing time sequence from runs $\mathrm{G}$ and $\mathrm{H}$ ).

In run $\mathrm{G}$ (bottom panels in fig. 12), the high- $l$ gas that accretes supersonically in the equatorial plane from large radii eventually tries to join the turbulent subsonic torus at small radii. The torus acts in this case as an obstacle with which the supersonic gas collides at about $120 R_{S}$ in the equatorial plane. There a strong shock forms. At smaller radii, the post-shock gas splits into two streams that flow around the torus. However, the split is not into two exactly equal halves. At the early phase of the evolution asymmetry is very small as it is due to random numerical asymmetry. However with time the asymmetry grows due

to strong shocks. In our simulations strong shocks are handled by artificial viscosity (Stone \& Norman 1982).

The asymmetry amplified by shocks propagate into other parts of the flow. In particular, the torus becomes asymmetric. Subsequently, a larger fraction of the equatorial inflow can flow in one of the hemisphere compared to the other hemisphere (Fig. 12, left bottom panel, illustrates a phase where more gas flows in the 'southern' hemisphere). Once above the torus, the equatorial inflow pushes the polar inflow away from the torus in the ' $z$ ' direction 
(seen as an oblique shock that oscillates and expands in the $z$ direction). The asymmetric interaction of the high- $l$ equatorial and low- $l$ polar inflows can be also seen in the angular momentum contours which are more compressed on one side of the torus. The green colored region in Fig. 12 expands and the density in the region at radii $\lesssim 40 R_{S}$ on the 'southern' side of the torus slightly decreases (Fig. 12, middle bottom panel). When this happens, the polar inflow of low-l matter starts to push the shock back toward the equator. The oblique shock swings and more of the equatorial inflow goes around the torus from the 'northern' side. The scenario repeats (Fig. 12, right bottom panel). As the oscillations continue, the oblique shock expands because matter has to flow around the constantly growing torus.

For $\gamma=1.01$, the torus is confined by the supersonic inflow. Therefore, asymmetry is strong only at small radii whereas at large radii the flow is symmetric as it is not affected by the presence of the asymmetric obstacle. Thus the low amplitude oscillations in the mass accretion rate curve are caused by the oscillating torus. The relevant time scale here is the dynamical time scale at the radii where the equatorial inflow is split into two streams. Fig. 13 presents a part of the mass accretion curve for model $\mathrm{G}$ in the later times of evolution to show the periodic variability. The time period between the peaks, corresponds to the dynamical time scale at radius of about $84 R_{S}$ that corresponds to the outer radius of the torus where the high-l gas splits up into two parts.

We also calculated a power spectrum using the mass accretion rate curve in the later times of evolution. The power spectrum shows broad peak, at frequency corresponding the dynamical time scale at radius of about $84 R_{S}$. The peak is broad because the torus increases in size with time, in particular the torus radius increases. We computed additional models for $\gamma=1.01$ with higher resolution in $\theta$ direction (400 and 800 points in $\theta$ angle). We found that oscillations appear to be independent of the grid resolution.

For $\gamma>1.01$, the flow behaves in a similar ways as that in run G. However, there is an additional complexity due to high gas pressure and mixing. For $\gamma>1.01$, the flow is less gravitationally bound than in run $\mathrm{G}$ and the torus is not as much confined by the supersonic inflow. Therefore, in runs with higher gas pressure the flow is subsonic in a relatively big region where mixing between low- and high- $l$ occurs. Because of the shock amplified asymmetry the mixing leads to large scale asymmetry (compare run $\mathrm{G}$ and $\mathrm{H}$ in Fig. 12).

As we mentioned above, for $\gamma=4 / 3$ and $\gamma=1.2$, we also observe occasional bursts in the $\dot{M}_{a}$ evolution (see e.g. Fig. 1). The mass accretion rate can significantly increase, because of mixing of the high- and low- $l$ gas at large radii. The flaring behavior of the mass accretion rate in models for $\gamma=4 / 3$ and $\gamma=1.2$ is caused by the torus movement in the ' $\mathrm{z}$ ' direction. Fig. 10 (panels corresponding to $\gamma=1.2$ case) captured a situation when the torus 
moved toward the southern hemisphere and partially blocked an inflow of low- $l$ gas in the polar funnel. Then this low-l gas is pushed toward equator. When the torus later swings toward the northern hemisphere, a channel for the low- $l$ widens one sees a flare in the mass accretion rate curve. The reason we do not observe strong flares for $\gamma=1.01$ is that there is no mixing of gas with high- and low-l at larger distances.

We find that the flow oscillates in the ' $z$ ' direction and is asymmetric with respect to the equatorial plane. Instabilities in the accretion disks were studied by many authors. Pulsational instability of quasi-adiabatic and quasi-inviscid accretion disks, was examined by in many studies (e.g., Kato 1978 Muchotrzeb-Czerny 1986; Kato et al. 1988; Okuda et al. 1993). Kato et al. (1978) found that the condition for pulsation instability depend on how viscosity changes during pulsation. In our simulation only artificial viscosity is introduced, which is small. Nevertheless this viscous proves to be important.

In summary, we find that the asymmetry of the flow is initially induced by the numerical effects, but the propagation and amplification of the asymmetry strongly depends on the physical conditions in the accretion flow. In the cases where the gas pressure is reduced because of low $\gamma$ the asymmetry is stronger. For higher $\gamma$, the gas pressure is larger and the information about the perturbation propagates faster. The gas pressure works against effects of shocks and tries to restore the symmetry. We explore effects of gas pressure to confirm its role in maintaining symmetry in the flow. To this end, we performed additional simulations for the same parameters as run J, but with higher $c_{\infty}$ corresponding to models with $R_{S}^{\prime}=10^{-2}$. Fig. 14 shows time evolution of velocity field of one of our exploratory runs. The flow with very high $c_{\infty}$ is symmetric as one would expect: the information of perturbances in high pressure gas propagates vary fast independently of grid effects. This simulations supports our reasoning that the asymmetry growing in the flow is not caused by numerical effects but rather by shocks amplifying initial seed asymmetry.

Our time dependent simulations occassionally show structures that resemble features of steady state disk solutions, such as backflows close to the equatorial plane (Kluzniak \& Kita, 2000, and our Fig. 14), or tori in the innermost part of the flow, whose angular momentum changes from sub- to super-Keplerian (Figs. 11, 12). Such tori may be relevant to high frequency QPOs (e.g. Blaes et al., 2007).

\section{Conclusions}

We report on next phase of our study of rotating accretion flows onto black holes. We consider hydrodynamical (HD) accretion flows with a spherically symmetric density 
distribution at the outer boundary but with spherical symmetry broken by the introduction of a small, latitude-dependent angular momentum. We study accretion flows by means of numerical two-dimensional, axisymmetric, HD simulations for variety of the adiabatic index, $\gamma$ and the gas temperature at infinity, $c_{\infty}$. Our work is an extension of work done by PB03a who consider models for only $\gamma=5 / 3$. We also reran some models from PB03a with higher resolution. The latter are fully consistent with lower resolution PB03a's runs.

Our main result is that the flow properties such as the topology of the sonic surface and time behavior strongly depend on $\gamma$ but little on $c_{\infty}$. In particular, for $1<\gamma<5 / 3$, the mass accretion rate shows large amplitude, slow time-variability which is a result of mixing between slow and fast rotating gas. This temporal behavior differs significantly from that in models with $\gamma \approx 5 / 3$ where the accretion rate is relatively constant and from that in models with $\gamma \approx 1$ where the accretion exhibits small amplitude quasi-periodic oscillations. The key parameter responsible for the differences is the sound speed of the accretion flow which in turn determines the strength of shocks and whether the flow is dominated but gas pressure $(\gamma \lesssim 5 / 3)$, radiation pressure $(1<\gamma<5 / 3)$, or rotation $(\gamma \gtrsim 1)$. Despite these differences, the time-averaged mass accretion rate in units of the corresponding Bondi rate is a weak function of $\gamma$ and $c_{\infty}$.

We realize that our simulations do not capture several physical processes that may be important in real systems (e.g., magnetic fields, radiative cooling and heating, energy dissipation). However, our simulations are done within a general framework so that some of these processes can be straightforwardly added (e.g., magnetic fields as in PB03b, or radiative processes as in Proga 2007). Therefore, this work could serve as a good reference point to analyze and interpret more complete and complex simulations (e.g., we already reran some of the models from this paper including magnetic fields, Moscibrodzka and Proga, in preparation).

ACKNOWLEDGMENTS: We thank Bozena Czerny and Marek Abramowicz for very useful comments. We acknowledge support provided by the Chandra award TM7-8008X issued by the Chandra X-Ray Observatory Center, which is operated by the Smithsonian Astrophysical Observatory for and on behalf of NASA under contract NAS8-39073. M.M also acknowledges supported in part by grant 1P03D 00829 of the Polish State Committee for Scientific Research (KBN). While D.P. acknowledges support from NASA under ATP grant NNG05GB68G. 


\section{REFERENCES}

Abramowicz, M.A., Chen, X., Kato, S., Lasota, J.-P., Regev, O. 1995, ApJ, 438, L37

Blandford, R.D., \& Begelman, M.C. 1999, MNRAS, 303, L1

Blaes O. M., Šrámková E., Abramowicz M.A., Kluźniak W., Torkelsson U.; 2007: Ap.J. vol. 665, pp. 642-653

Chen, X., Taam, R.E., Abramowicz, M.A., \& Igumenshchev, I.V. 1997, MNRAS, 285, 439

Clarke, D., Karpik, S., \& Henriksen, R.N. 1985, ApJS, 58, 81

Done C., Gierlinski M., Kubota A., 2007, A\&ARev, temp, 3D

Hawley, J.F., Smarr, L.L., \& Wilson, J.R. 1984a, ApJ, 277, 296

Hawley, J.F., Smarr, L.L., \& Wilson, J.R. 1984b, ApJS, 55, 211

Ichimaru, S. 1977, ApJ, 214, 840.

Janiuk A., Proga D., 2007, arXiv0708.2711

Kato S., 1978, MNRAS, 185, 629

Kato S., Honma F., Matsumoto R., 1988, MNRAS, 231, 37 Krolik J., 1999,

Kluźniak W., Kita D., 2000, astro-ph/0006266

Mészáros, P., 2006, Rep. Prog. Phys., 69, 2259-2322

Mo H.J., Mao S., White S. D. M, 1998, MNRAS, 295, 319

Muchotrzeb-Czerny B., 1986, AcA, 36, M1

Proga D., Begelman M., 2003, ApJ, 582, 69 (PB03a)

Proga D., Begelman M., 2003b, ApJ, 592, 767 (PB03b)

Narayan, R., \& Yi, I. 1994, ApJ, 428, L13

Narayan, R., \& Yi, I. 1995, ApJ, 444, 231

Okuda T., Mineshinge S., 1993, Astrophysics and Space Science, 210, 361

Paczyński, B., \& Wiita, J. 1980, A\&A, 88, 23

Proga D., 2007, ApJ, 661, 693 
Sharma, P., Quataert, E., Hammett, G., \& Stone, J. M. 2007, ApJ, 667, 714

Rees, M.J., Begelman, M.C., Blandford, R.D., \& Phinney, E.S. 1982, Nature, 295, 17

Stone, J.M., \& Norman, M.L. 1992, ApJS, 80, 753 
Table 1: Summary of parameter survey.

\begin{tabular}{|c|c|c|c|c|c|c|c|}
\hline Run & $\begin{array}{l}\text { Radial } \\
\text { resolution }\end{array}$ & $R_{S}^{\prime}$ & $R_{C}^{\prime}$ & $\gamma$ & $t_{f}$ & $l_{a}^{\max }$ & $\dot{M}_{a} / \dot{M}_{B}$ \\
\hline A & 220 & $10^{-5}$ & $5 \times 10^{-4}$ & 1.01 & $1.97 \times 10^{4}$ & 0.77 & 0.45 \\
\hline B & 220 & $10^{-5}$ & $5 \times 10^{-4}$ & $4 / 3$ & $3.10 \times 10^{4}$ & 0.9 & 0.25 \\
\hline $\mathrm{C}$ & 220 & $10^{-5}$ & $5 \times 10^{-4}$ & $5 / 3$ & $3.14 \times 10^{4}$ & 0.92 & 0.13 \\
\hline $\mathrm{D}$ & 180 & $10^{-4}$ & $5 \times 10^{-3}$ & 1.01 & $2.80 \times 10^{4}$ & 0.82 & 0.40 \\
\hline $\mathrm{E}$ & 180 & $10^{-4}$ & $5 \times 10^{-3}$ & $4 / 3$ & $2.75 \times 10^{4}$ & 0.85 & 0.13 \\
\hline $\mathrm{F}$ & 180 & $10^{-4}$ & $5 \times 10^{-3}$ & $5 / 3$ & $3.19 \times 10^{4}$ & 0.87 & 0.15 \\
\hline G & 140 & $10^{-3}$ & $5 \times 10^{-2}$ & 1.01 & $3.48 \times 10^{4}$ & 0.9 & 0.30 \\
\hline $\mathrm{H}$ & 140 & $10^{-3}$ & $5 \times 10^{-2}$ & 1.2 & $3.10 \times 10^{4}$ & 0.94 & 0.20 \\
\hline I & 140 & $10^{-3}$ & $5 \times 10^{-2}$ & $4 / 3$ & $3.56 \times 10^{4}$ & 0.9 & 0.17 \\
\hline $\mathrm{J}$ & 140 & $10^{-3}$ & $5 \times 10^{-2}$ & $5 / 3$ & $2.67 \times 10^{4}$ & 0.87 & 0.30 \\
\hline
\end{tabular}




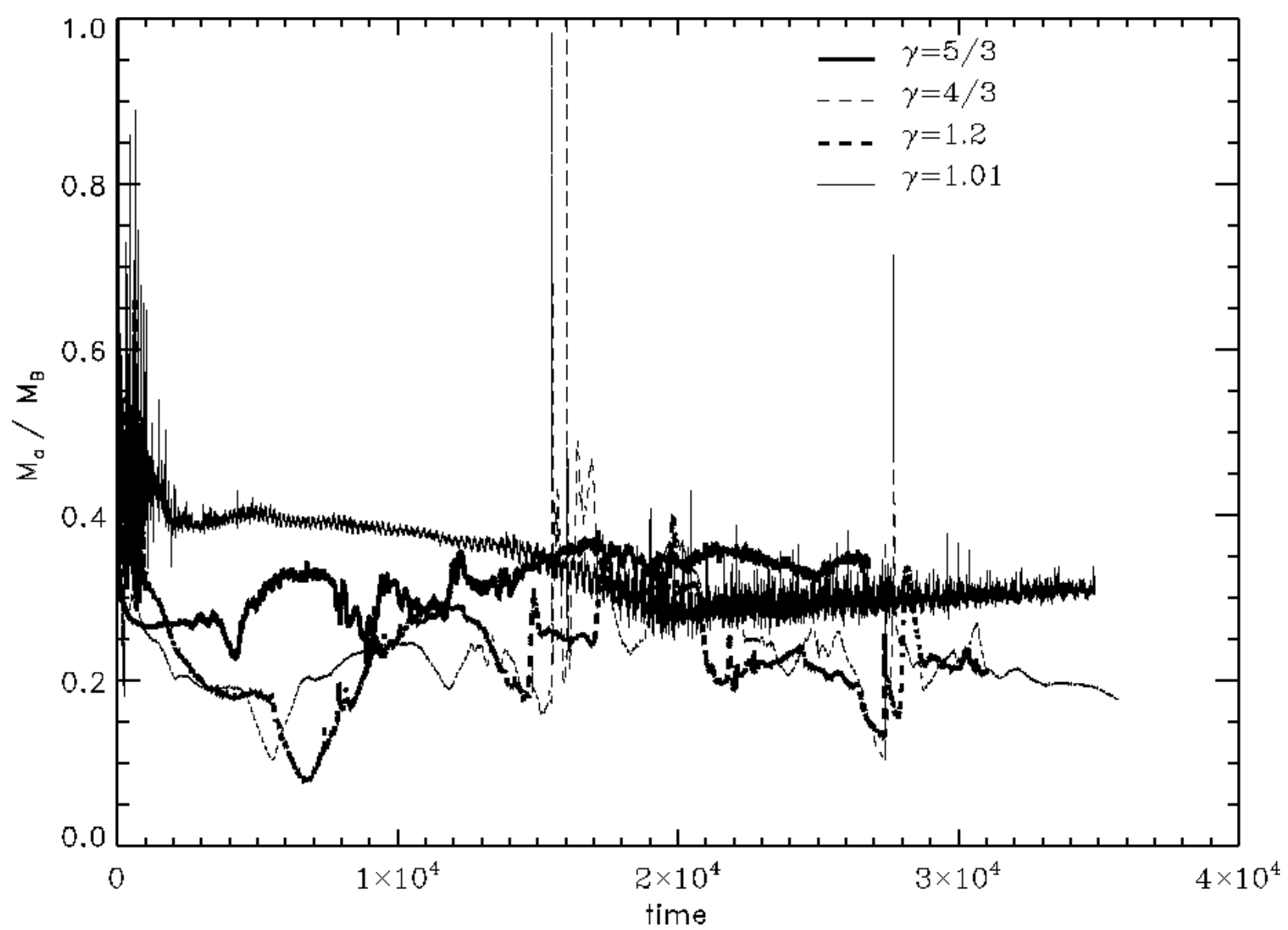

Fig. 1.- Mass accretion rate as a function of time for $R_{S}^{\prime}=10^{-3}$. Time is given in the units of dynamical time at the inner boundary. Note the strong flaring behavior for $\gamma=1.2$, and $4 / 3$. 


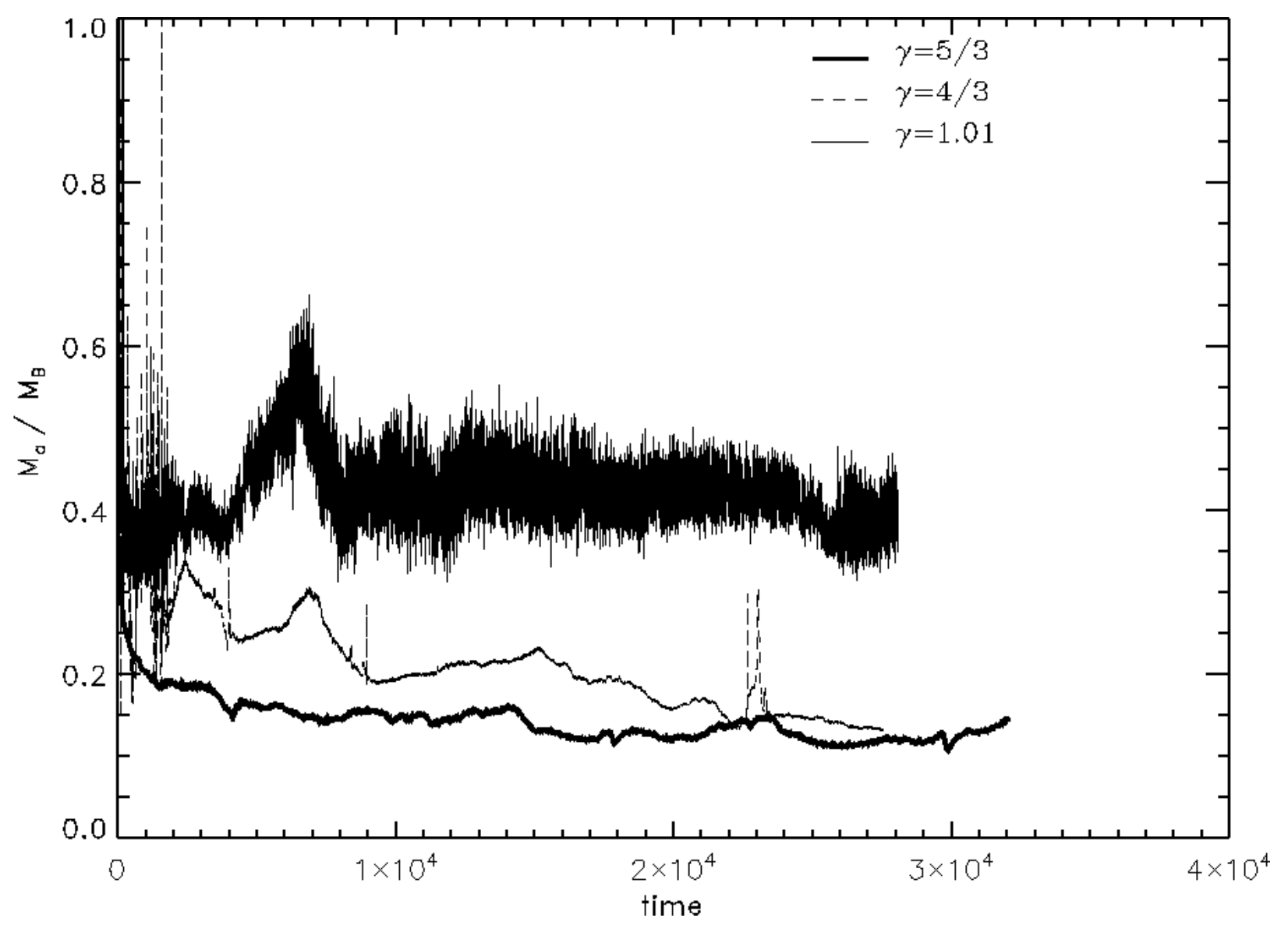

Fig. 2.- As in Fig. 1 but for $R_{S}^{\prime}=10^{-4}$. 


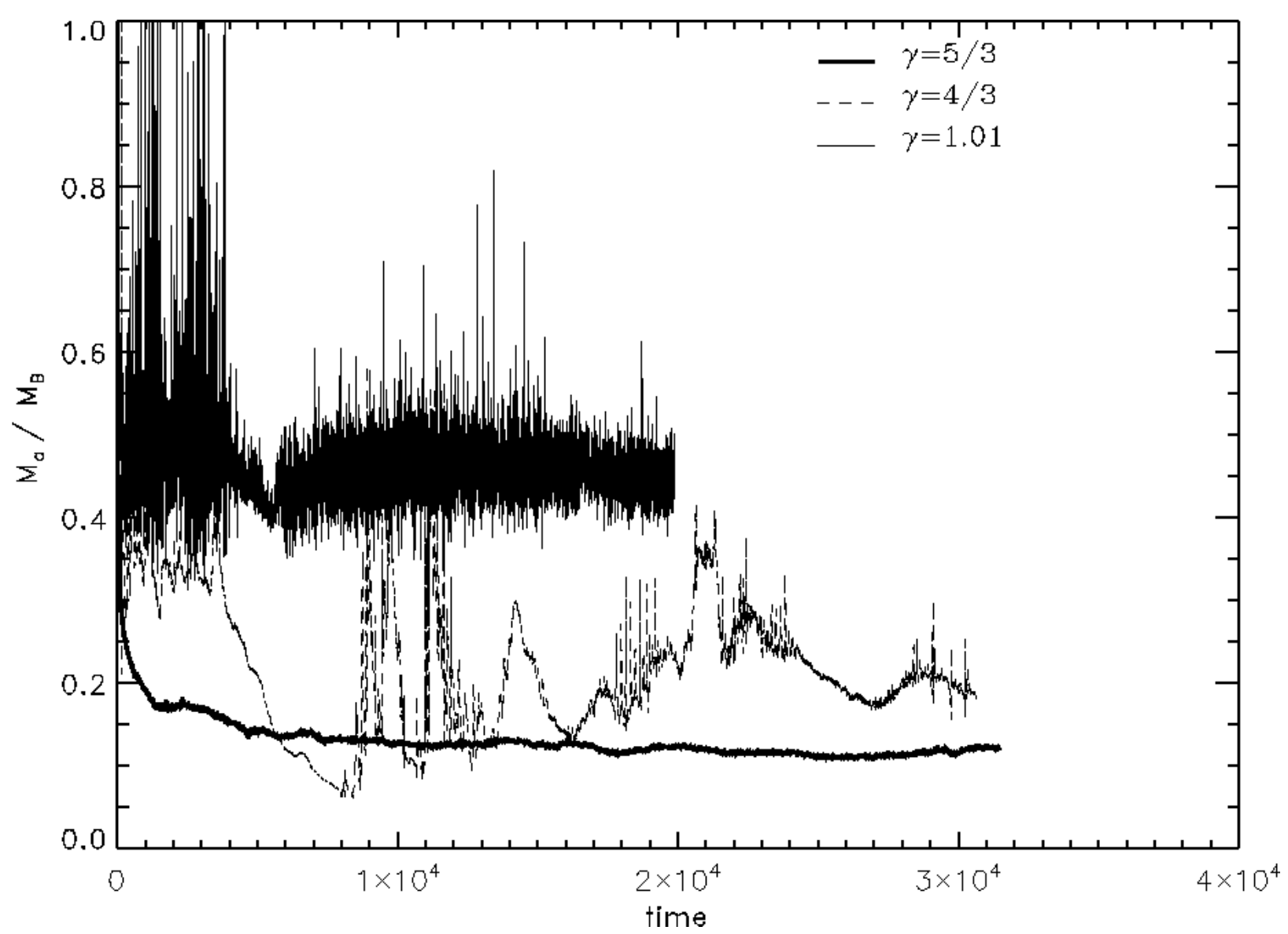

Fig. 3.- As in Fig. 1 but $R_{S}^{\prime}=10^{-5}$. 


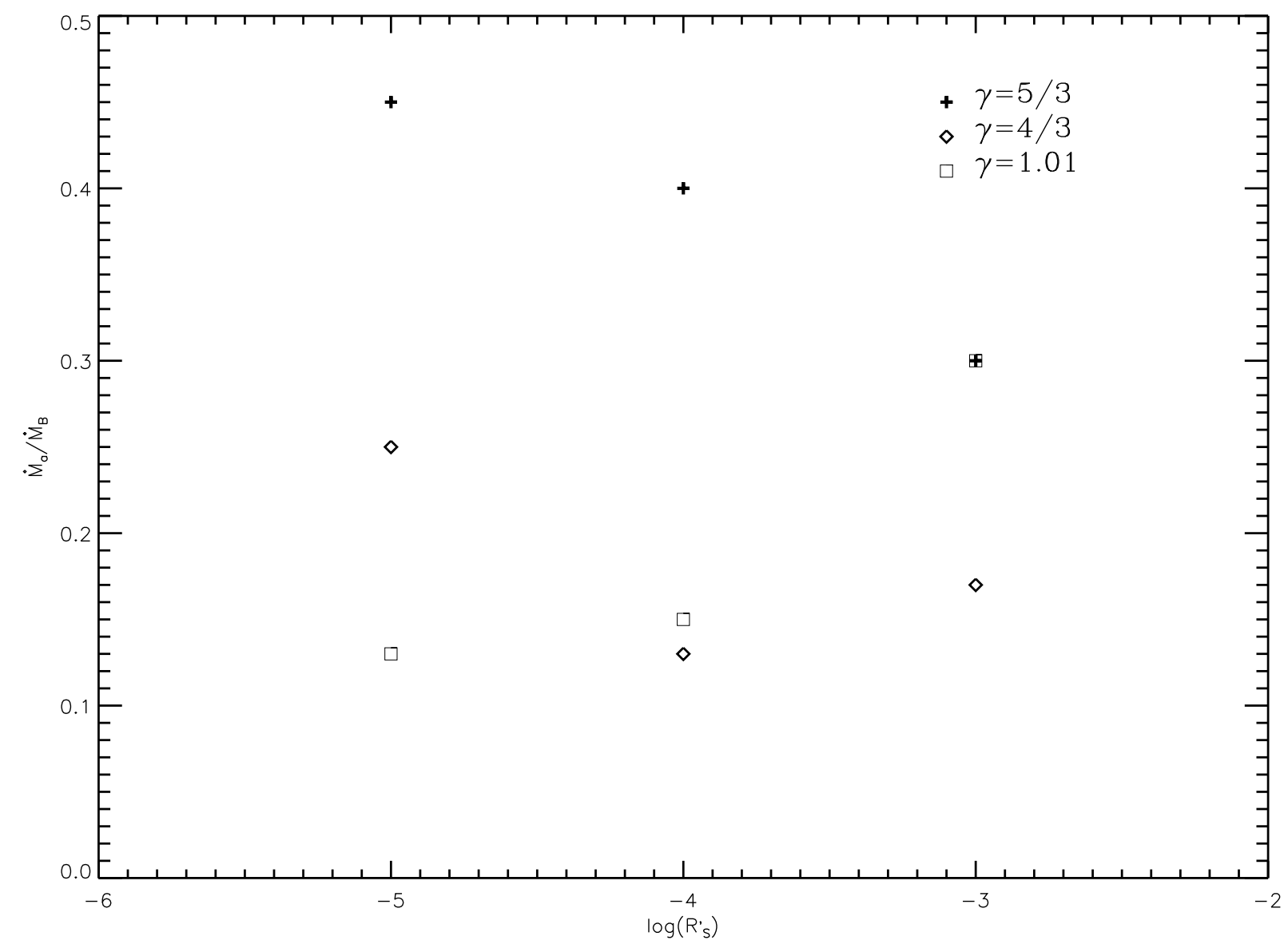

Fig. 4.- Mass accretion rate at the end of the simulations as a function of $R_{S}^{\prime}$ for different $\gamma$. 

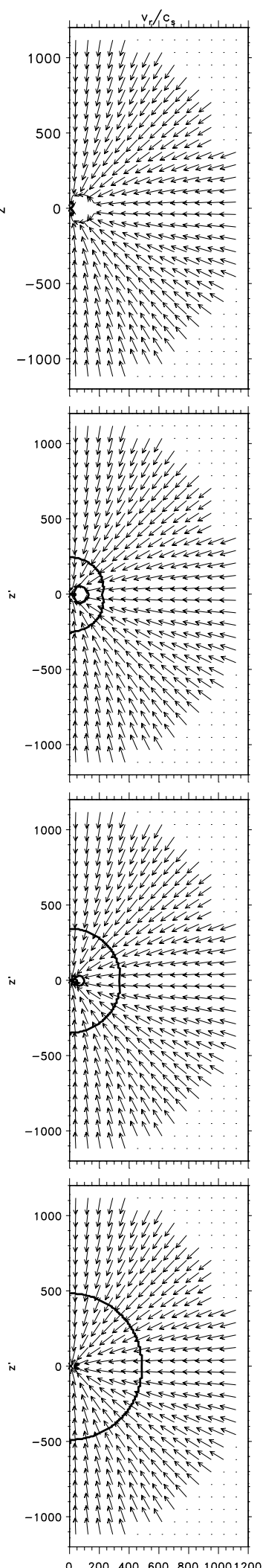
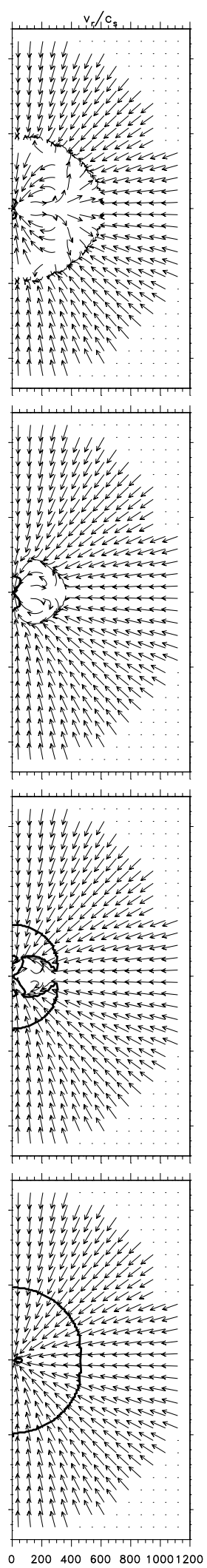
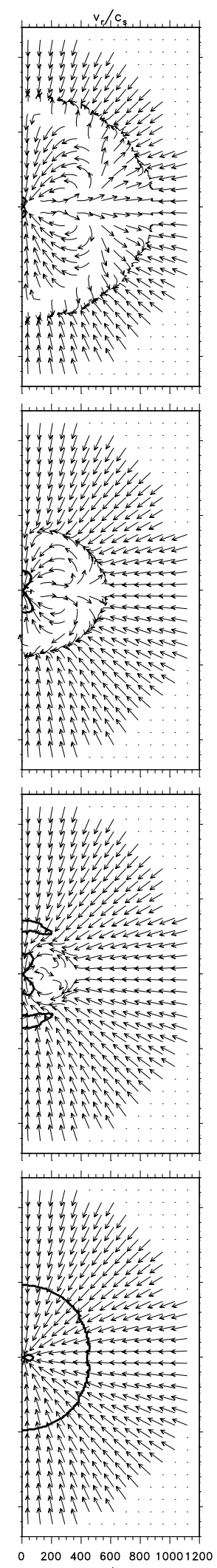
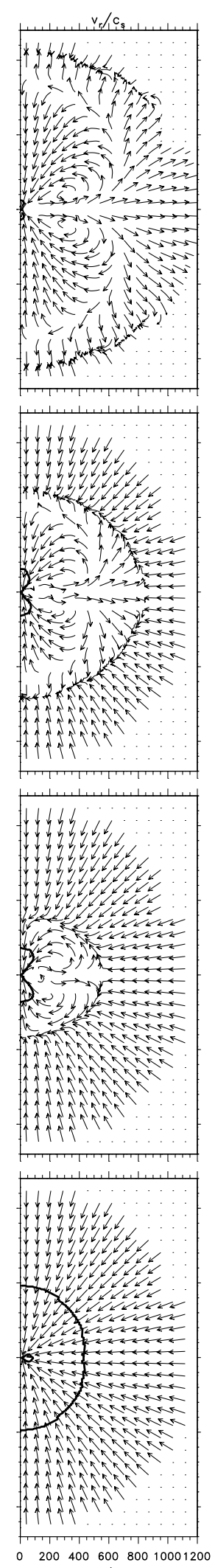
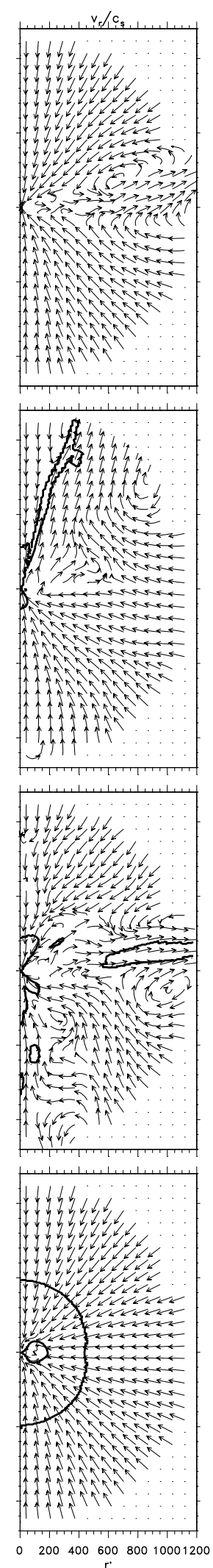

Fig. 5. - Sequences of poloidal velocity fields, and sonic surface at five different times (from left to right $\mathrm{t}=177.3,701.0,1169.1,1754.3$, and $t_{f}$ respectively, in units of dynamical time at inner radius of computational domain) for $\gamma=5 / 3,4 / 3,1.2$, and 1.01 (from top to bottom). 

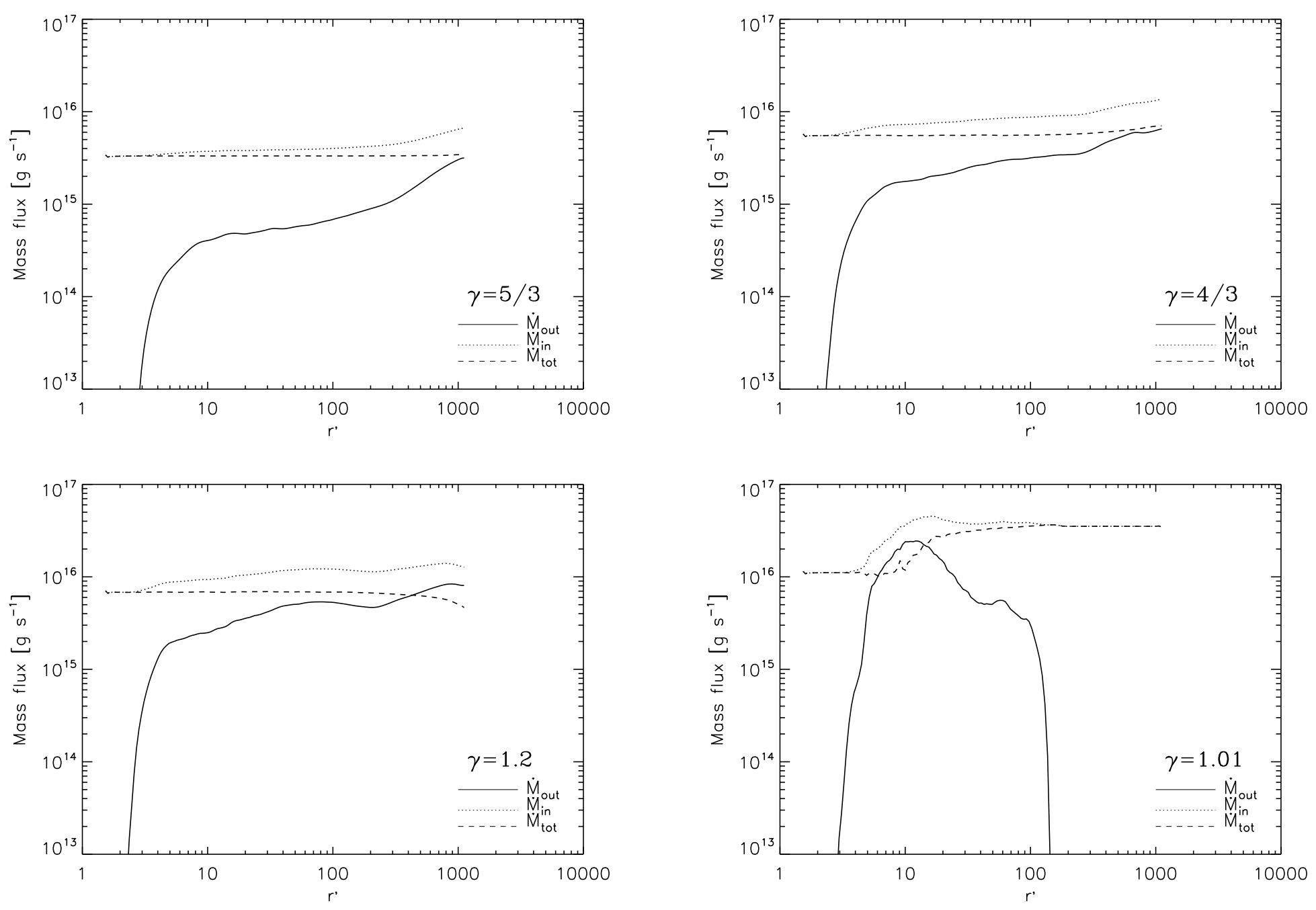

Fig. 6.- Mass flux rates as functions of radius for models with $R_{S}^{\prime}=10^{-3}$ and different $\gamma$ time-averaged at the end the simulations. For $\gamma=5 / 3,4 / 3$, and 1.2 , the average is taken over $\Delta t=1.75 \times 10^{4}-t_{f}$ time period. For $\gamma=1.01$, we average over $\Delta t=2.5 \times 10^{4}-t_{f}$. 

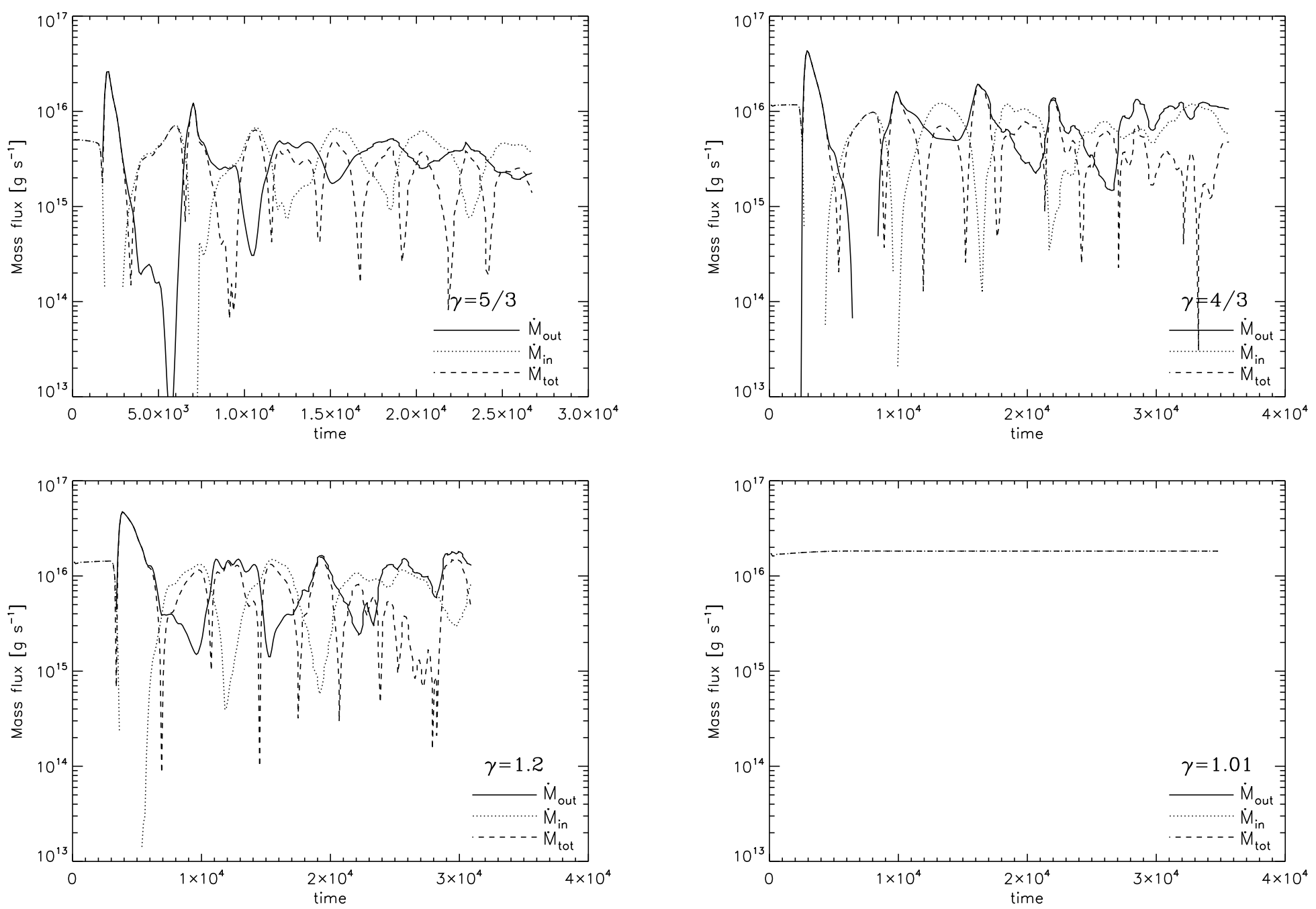

Fig. 7.- Mass flow rates at the outer boundary as functions of time for models with $R_{S}^{\prime}=10^{-3}$ and different $\gamma$. 

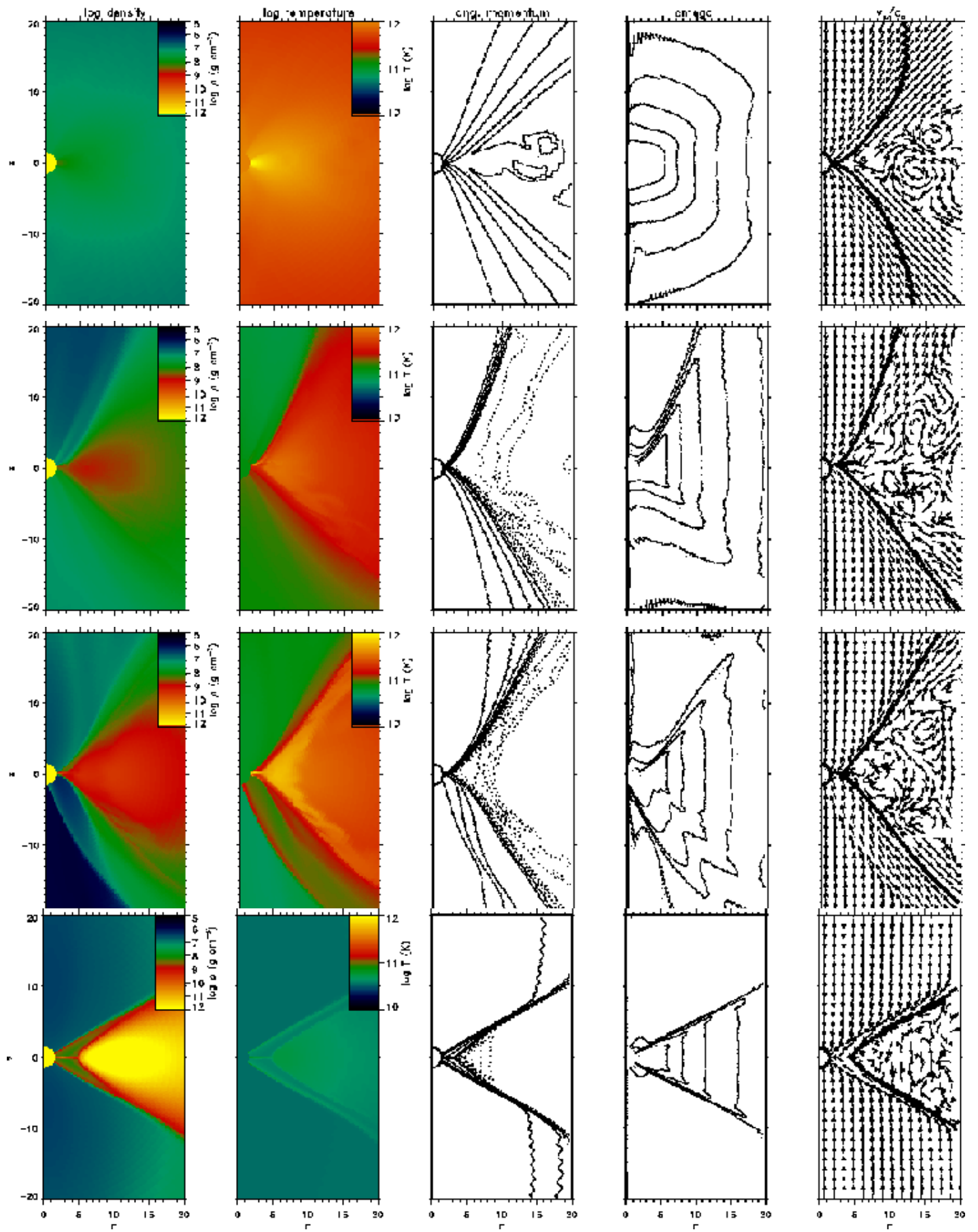

Fig. 8.- Two-dimensional structure of various quantities at the last time step for $\gamma($ $\gamma$ index 5/3, 4/3, 1.2, and 1.01 from top to bottom). The panels from left to right are snapshots of $\log \rho, \log T, l, \log \Omega$, and of the fluid poloidal velocity direction overplot with sonic surface contour. We do not draw labels for angular momentum and angular velocity because of strong compression of contours. The angular momentum contours are between 0.2 and 1.1 with the step of 0.1 . The contorus for angular velocity are between -2.5 and -1.5 with the step of 0.25 . The scale of the figure is $20 R_{S}$. 


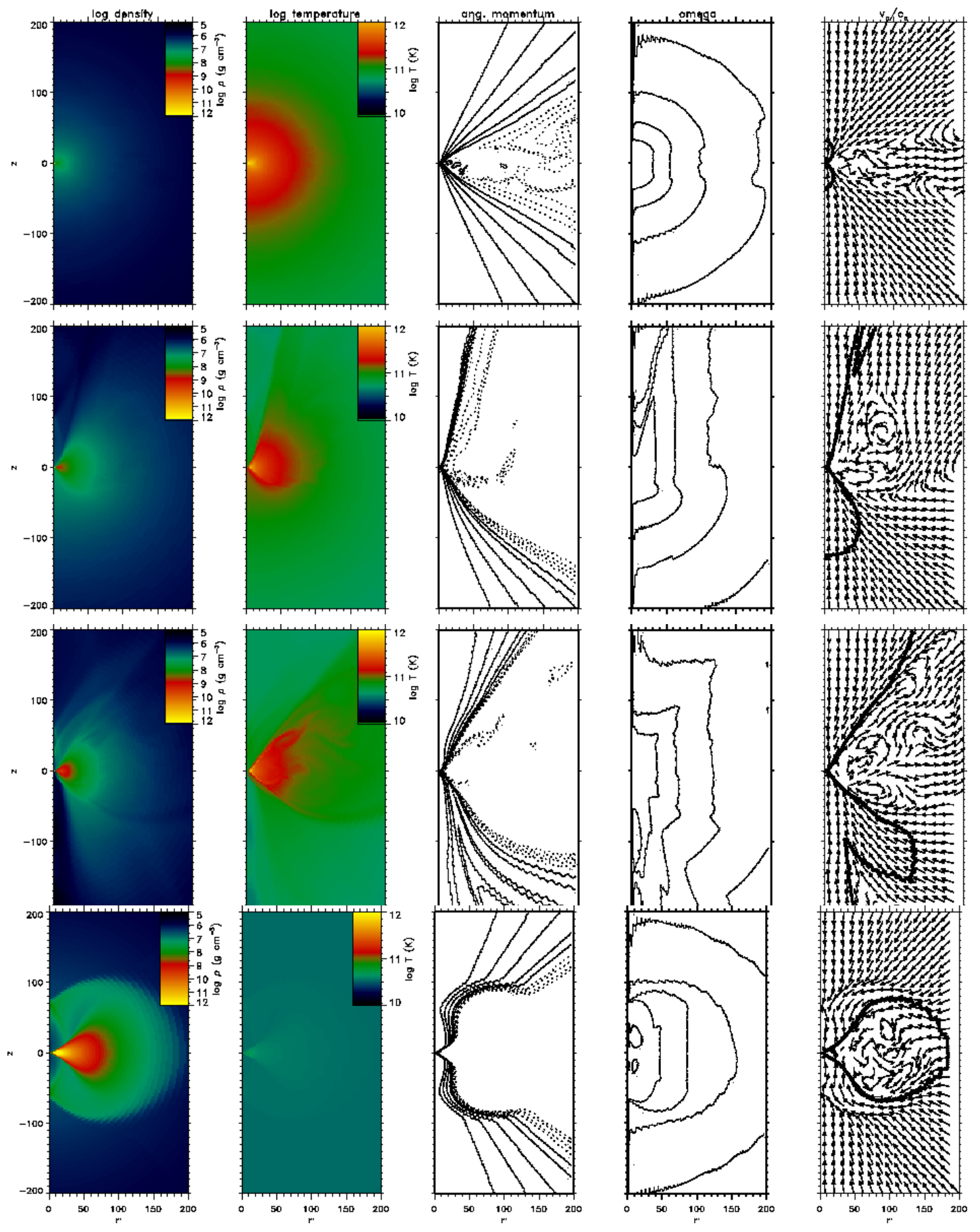

Fig. 9.- As in Fig. 8 but on the scale of $200 R_{S}$. The $l$ contours are between 0.2 and 1.1 with step of 0.1 while the $\log \Omega$ contours are between -5.5 and -3.0 with step of 0.5 . 

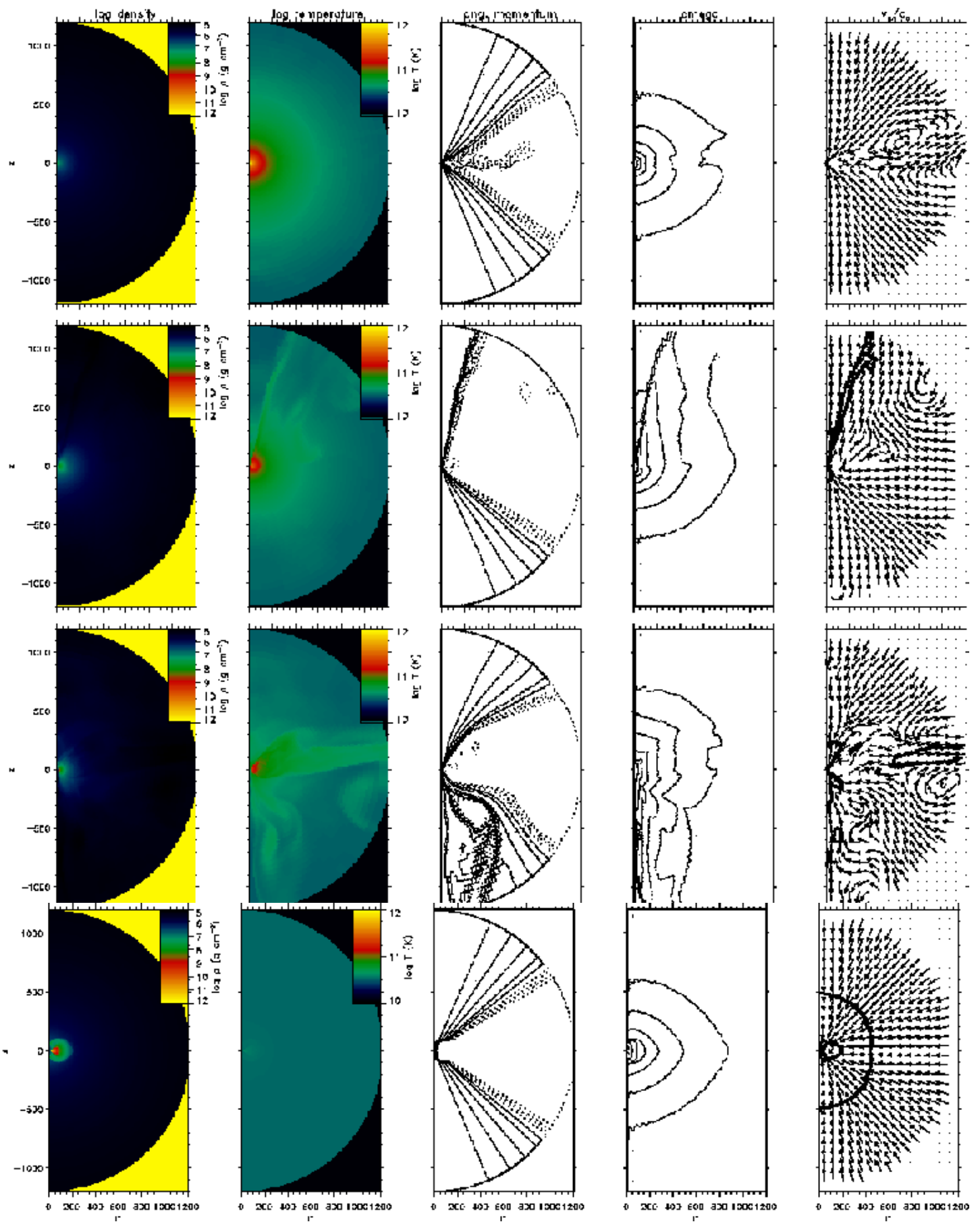

Fig. 10. - As in Fig. 8 but on the scale of $1200 R_{S}$ (showing the whole computational domain). The $l$ contours are between 0.2 and 1.1 with step of 0.1 . The log $\Omega$ contours are between -5.5 and -3.0 with step of 0.5 . 


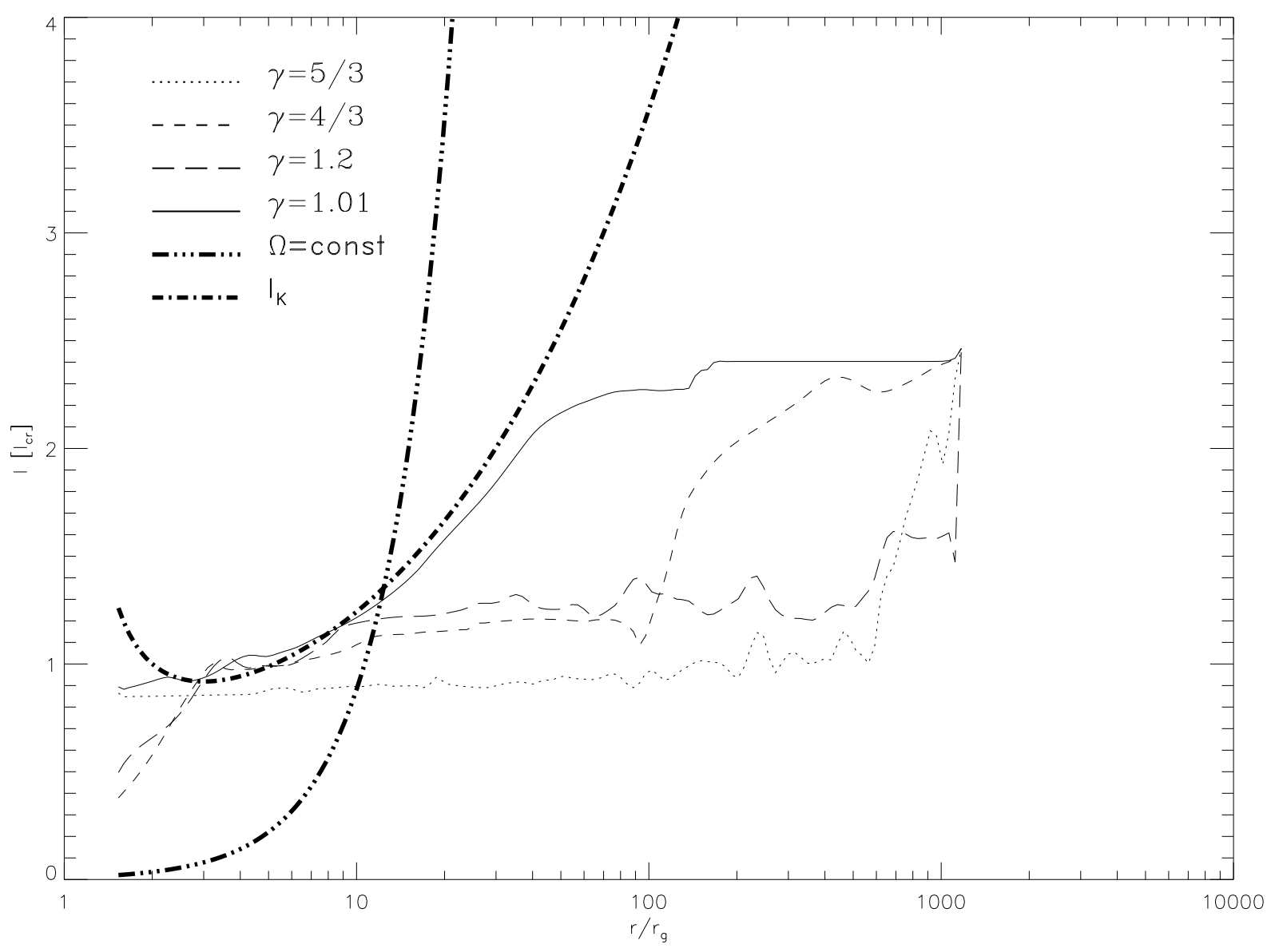

Fig. 11. - Radial profile of angular momentum along the equatorial plane for different $\gamma$ at the end of simulations. For comparison Keplerian angular momentum and angular momentum corresponding to constant angular velocity are shown, too. 


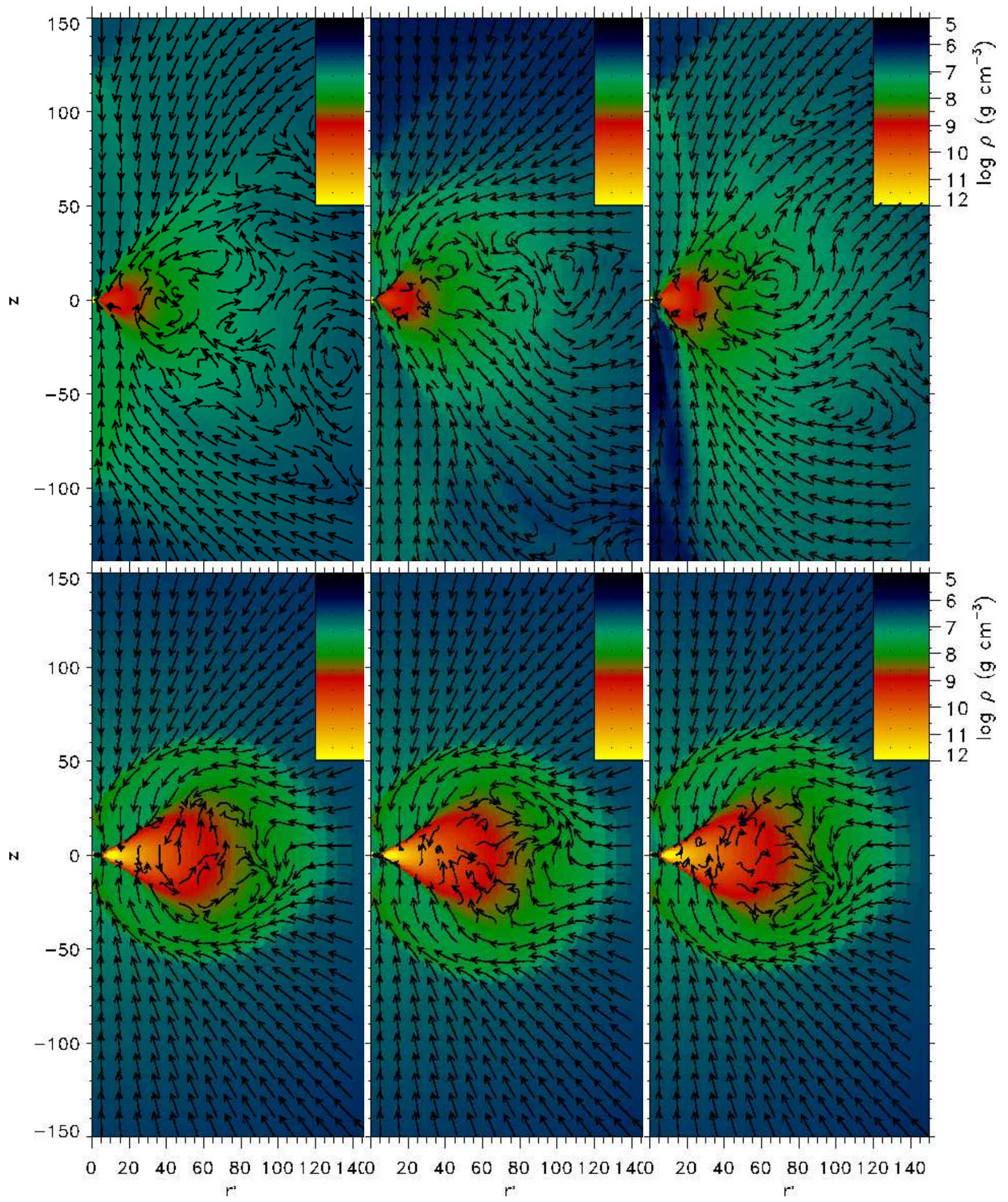

Fig. 12.- Sequence of density maps overplotted by the direction of the poloidal velocity for $\gamma=1.2$ (upper panels) and $\gamma=1.01$ (lower panels) for times $\mathrm{t}=1.97 \times 10^{4}, 2.1 \times 10^{4}$, and $2.8 \times 10^{4}$ (upper panels) and for $\mathrm{t}=9.7 \times 10^{3}, 1.12 \times 10^{4}$, and $1.24 \times 10^{4}$ (lower panels). These time sequences illustrate the evolution of oblique shocks forming in the infalling gas in the polar funnel caused by the torus. 


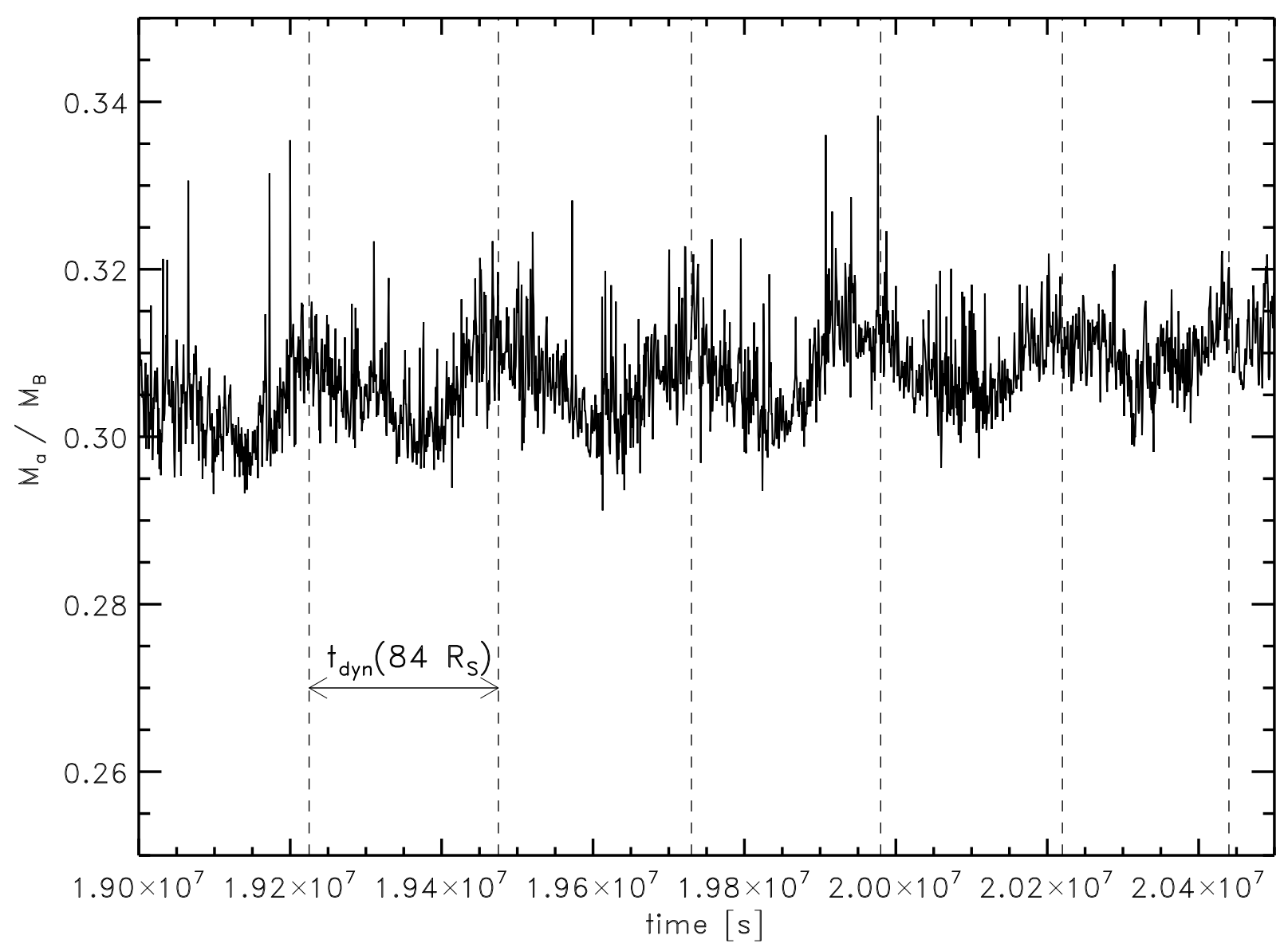

Fig. 13.- Mass accretion rate curve for model $G$ in the later time of evolution. The curve shows a periodic variability with the period corresponding to the dynamical time scale at radius of about $84 R_{S}$, where we observe the strongest oscillations the torus and asymmetry in the flow (see the lower panels in Fig. 12.) 

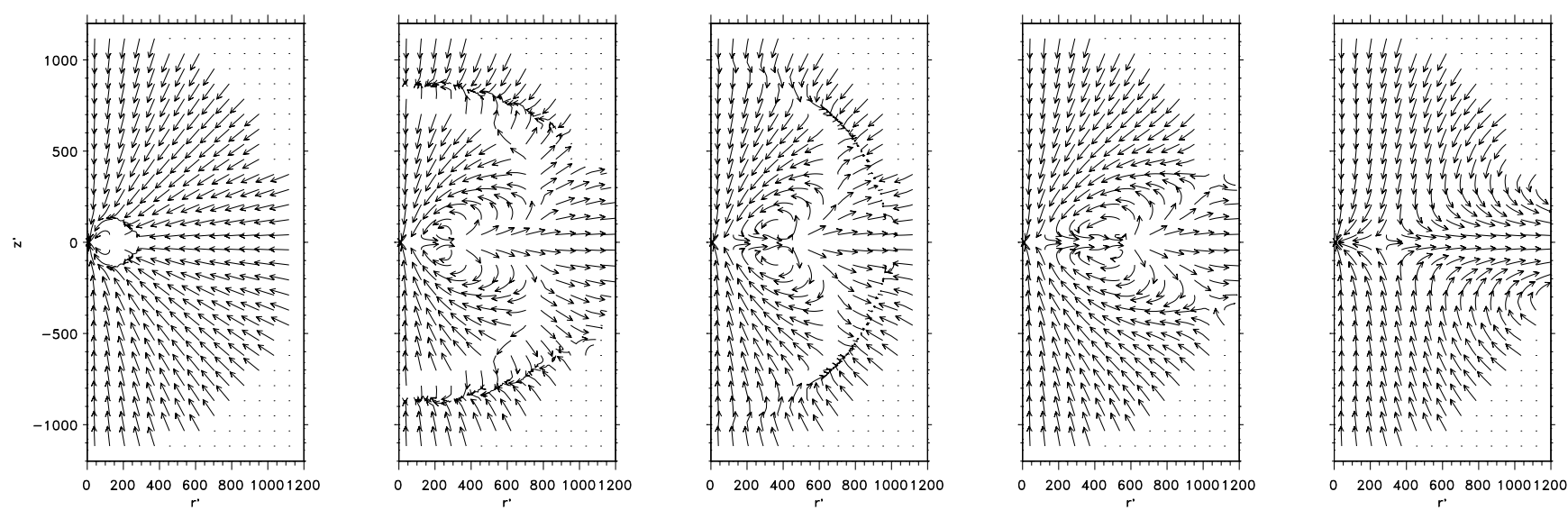

Fig. 14. - Sequence of poloidal velocity fields for five different time for with $R_{S}^{\prime}=10^{-2}$. The computational domain and initial condition are the same as in J model, except that the $c_{s, \infty}$ is much higher. 